
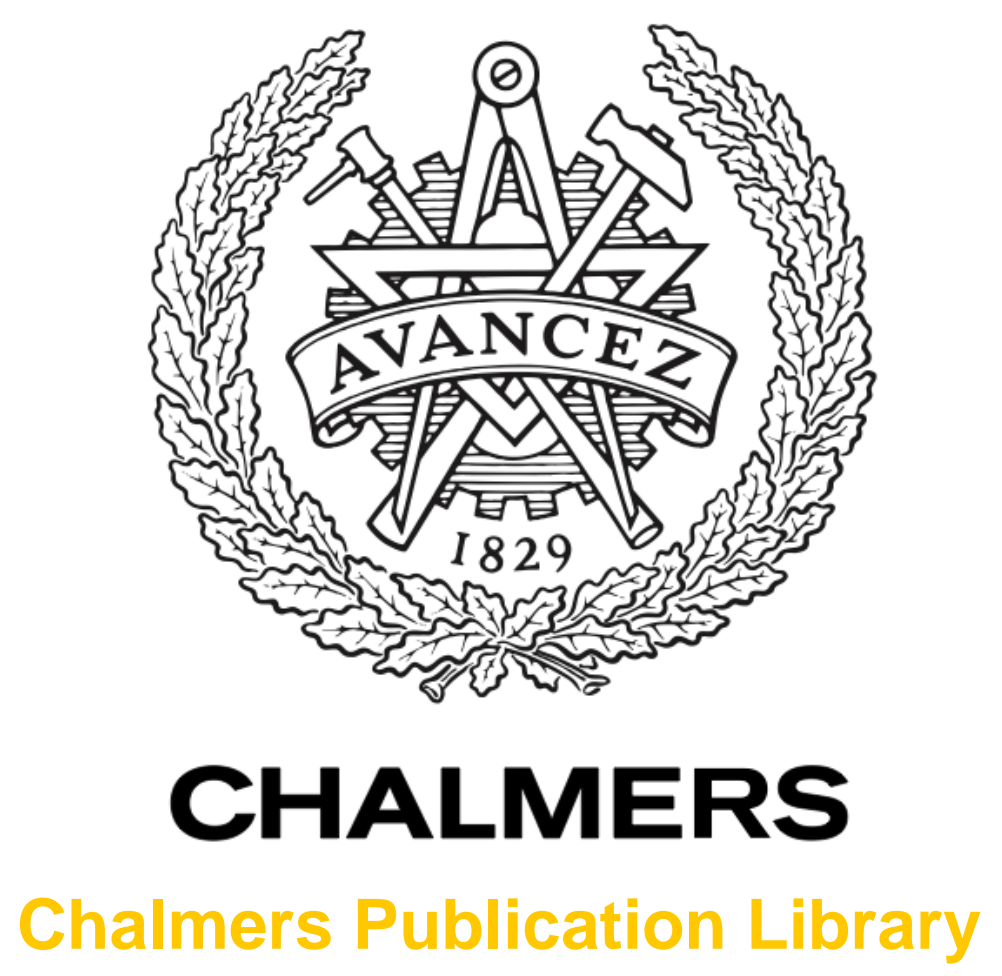

Silicon Integrated InGaAs/InAIAs/AIAs HBV Frequency Tripler

This document has been downloaded from Chalmers Publication Library (CPL). It is the author's version of a work that was accepted for publication in:

IEEE Electron Device Letters (ISSN: 0741-3106)

Citation for the published paper:

Malko, A. ; Bryllert, T. ; Vukusic, J. (2013) "Silicon Integrated InGaAs/InAlAs/AlAs HBV

Frequency Tripler". IEEE Electron Device Letters, vol. 34(7), pp. 843 - 845.

http://dx.doi.org/10.1109/LED.2013.2262131

Downloaded from: http://publications.lib.chalmers.se/publication/179676

Notice: Changes introduced as a result of publishing processes such as copy-editing and formatting may not be reflected in this document. For a definitive version of this work, please refer to the published source. Please note that access to the published version might require a subscription. 


\title{
Silicon Integrated InGaAs/InAlAs/AlAs HBV Frequency Tripler
}

\author{
Aleksandra Malko, Student Member, IEEE, Tomas Bryllert, Member, IEEE, Josip Vukusic, \\ Member, IEEE and Jan Stake, Senior Member, IEEE
}

\begin{abstract}
We present an integrated heterostructure barrier varactor (HBV) frequency tripler on silicon substrate. The InGaAs/InAlAs/ AlAs material structure was transferred onto the silicon wafer using low temperature plasma assisted bonding. The presented multiplier operates in the $\mathrm{W}$-band $(90-110 \mathrm{GHz})$. The module delivers $22.6 \mathrm{dBm}$, with a conversion loss of $6 \mathrm{~dB}$, and $9 \%$ 3-dB bandwidth.
\end{abstract}

Index Terms - Frequency multipliers, heterostructure barrier varactors, heterogeneous integration, integrated circuits, millimeter-wave diodes, silicon, wafer bonding, III-V semiconductors.

\section{INTRODUCTION}

$\mathrm{T}$ HE III-V compound semiconductors are broadly used in active devices operating at $\mathrm{mm}$-wave and $\mathrm{THz}$ frequencies [1]- [2]. III-Vs offer high electron mobility, high bandgap and the epitaxial growth allows for fabrication of complex layer structures. However, at $\mathrm{THz}$ frequencies, monolithic integrated circuits require ultra thin substrates and on chip antenna probes for signal coupling, which occupy large wafer area. This is not easily made on materials like InP or GaAs which are fragile, expensive and are limited to small wafer sizes.

Silicon is a mechanically robust material. It is cheap, supports large wafer sizes $(>8 ")$, and is suitable for micromachining of complex $3 \mathrm{D}$ structures [3]. In addition, it has two times higher thermal conductivity than InP or GaAs, which is an advantage for power dissipating devices. By heterogeneous integration of III-Vs on $\mathrm{Si}$ high frequency active devices on membranes, integrated in waveguides will be possible [4]- [5]. This will provide an additional degree of freedom in the circuit design and fabrication.

Both direct epitaxial growth and epitaxial transfer methods have been used for the heterogeneous integration of III-Vs on $\mathrm{Si}$ [6]. For microwave applications a GaAs MESFET [7], an InGaP/ GaAs [8], InGaAs/ InP [9] HBTs, and an AlGaN/ GaN

Manuscript received January 29, 2013; accepted May 04, 2013. This work was supported by the Swedish Research Council (VR).

A. Malko is with the Terahertz and Millimetre Wave Laboratory, Department of Microtechnology and Nanoscience, Chalmers University of Technology, Göteborg, Sweden, (e-mail: malko@chalmers.se).

T. Bryllert, J. Vukusic and J. Stake are with the Terahertz and Millimetre Wave Laboratory, Department of Microtechnology and Nanoscience, Chalmers University of Technology, Göteborg, Sweden and with Wasa Millimeter Wave AB
HEMT [10] on Si have been demonstrated. Epitaxial transfer methods for $\mathrm{THz}$ frequency multipliers onto quartz [11]- [12] and AIN have been presented. However, direct integration of these integrated circuits onto $\mathrm{Si}$, and the studies of the thermal properties and losses have not been shown.

In this letter, an integrated InGaAs/ InAlAs/ AlAs HBV frequency tripler on silicon substrate is presented. Due to $55 \%$ thermal expansion coefficient mismatch between InP and $\mathrm{Si}$ a low temperature plasma assisted bonding was utilized [13]. The performance of the reported device is comparable with the state-of-the-art devices grown and processed directly on the lattice matched InP substrate [14]- [15]. This motivates further research, and development of applications operating at $\mathrm{THz}$ frequencies utilizing heterogeneous integrated III-Vs on silicon.

\section{DESIGN AND FABRICATION}

\section{A. HBV Devices}

The HBV is a semiconductor device, which under an applied voltage exhibits a nonlinear and symmetric capacitance [16]. The capacitance modulation is possible by combination of low/ high/ low bandgap material Fig.1. The barrier thickness is optimized for minimum leakage current and maximum breakdown voltage [17]. The device area and the number of barriers are designed to handle ca $1 \mathrm{~W}$ of the input power [18]. The HBV material structure was grown on 3 " InP substrate by molecular beam epitaxy. The HBV material was then transferred onto high resistivity silicon $(>10 \mathrm{k} \Omega \mathrm{cm})$ using LT plasma assisted wafer bonding [19]. In Fig. 2, a TEM image of the transferred material and the amorphous oxide layer at the bonded interface are shown.

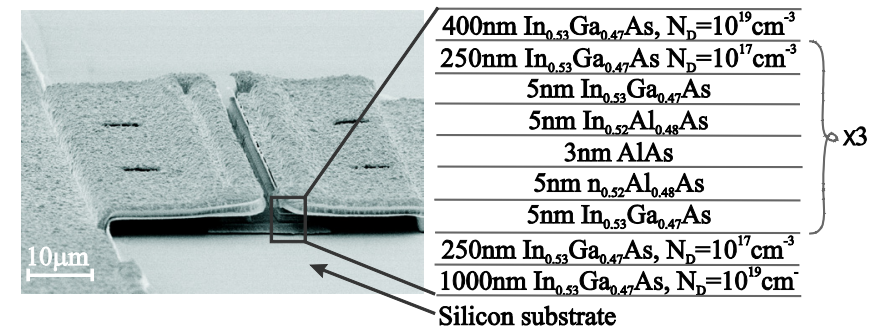

Fig.1. Left: SEM image of an integrated HBV diode with total 6 barriers, on the silicon substrate with the air bridge connections to the embedding circuit.

Right: depicted epitaxial layers of the HBV device. The mesa contact area is $7 \times 100 \mu \mathrm{m}^{2}$. 


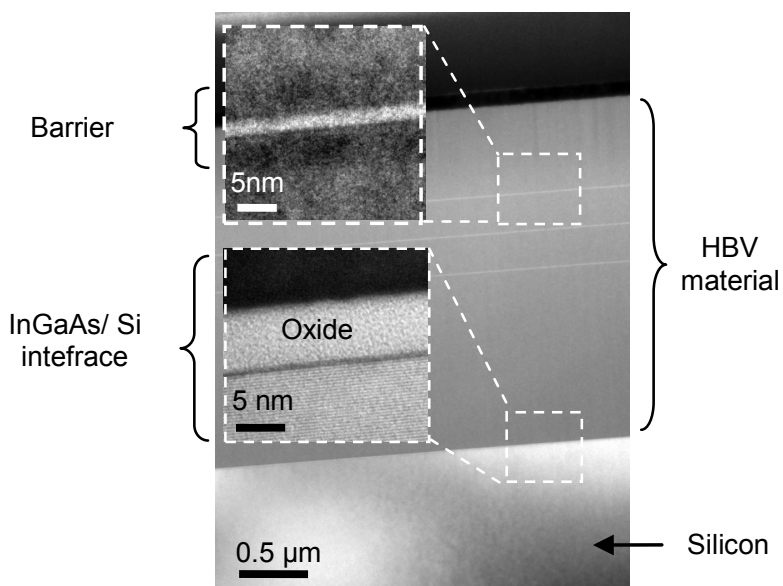

Fig. 2. TEM image presenting the III-V HBV on silicon, and the InGaAs$\mathrm{Si}$ interface, with $5 \mathrm{~nm}$ thick oxide layer.

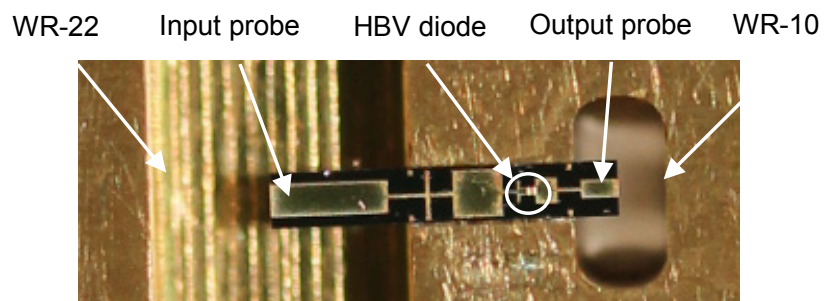

Fig. 3. Image of a silicon integrated HBV frequency tripler assambled in the waveguide channel. The chip dimensions are: $4.7 \mathrm{~mm} \times 0.8 \mathrm{~mm} \times 0.08 \mathrm{~mm}$ (length $\times$ width $\times$ thickness).

Subsequently, the wafers were diced into $20 \times 20 \mathrm{~mm}^{2}$ chips, cleaned, and the integrated diodes and circuits were fabricated. This process consists of standard III-Vs fabrication steps. In addition, a $100 \mathrm{~nm}$ thick $\mathrm{SiO}_{2}$ layer was sputtered on the silicon surface. The $\mathrm{SiO}_{2}$ passivation had no influence on the circuits RF performance, but reduced the substrate DC conductance, allowing for accurate I-V characterization of the active devices.

\section{B. Circuit}

The tripler circuit design is described in [15], [20]. An image of the silicon integrated frequency tripler assembled in the waveguide channel is shown in Fig. 3. The input signal is coupled to the circuit with a waveguide probe. The matching is realized in microstrip technology. The output waveguide will effectively block the fundamental harmonic $\left(\omega_{0}\right)$. The generated signal at $3 \omega_{0}$, is coupled to the output waveguide (WR-10) with a waveguide probe.

\section{RESULTS}

The input signal was generated with an Agilent E8257D signal generator. This signal was amplified with a Spacek Labs Ka-band power amplifier. A $10 \mathrm{~dB}$ directional coupler and an Agilent E4418B power sensor were used for accurate control of the available pump power. The output power at the third harmonic was measured with an Erickson PM4 power meter. The characterization was performed for an input frequency sweep from $30-34 \mathrm{GHz}$ with a $0.2 \mathrm{GHz}$ step. The input power drive level was in the $20-29 \mathrm{dBm}$ range.

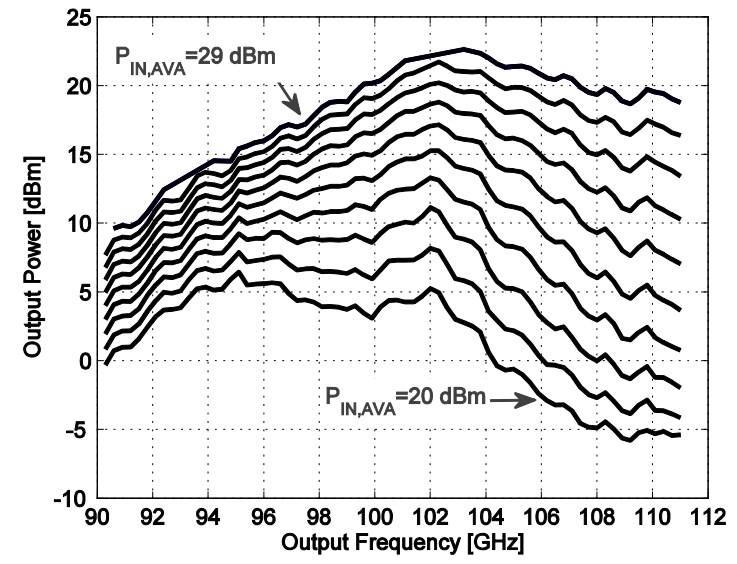

Fig. 4. Measured output power versus output frequency as a function of the available input power from $20-29 \mathrm{dBm}$ in steps of $1 \mathrm{dBm}$.

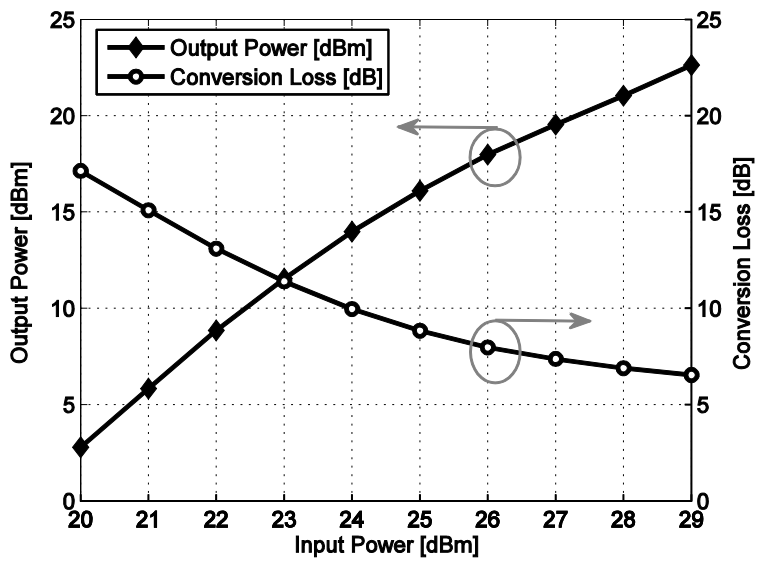

Fig. 5. Output power and conversion loss as a function of input power at $103 \mathrm{GHz}$ output frequency.

The RF performance of the demonstrated $\times 3$ frequency multiplier is shown in Fig. 4. The maximum output power for $29 \mathrm{dBm}$ of the input power measured at $103 \mathrm{GHz}$ was $22.6 \mathrm{dBm}(184 \mathrm{~mW})$. The $3-\mathrm{dB}$ bandwidth for the presented device was approximately $9 \%$. In Fig. 5 . the output power and conversion loss collected at the center frequency are presented. The conversion loss for this device is about $6 \mathrm{~dB}$, and corresponds to $23 \%$ efficiency.

Due to the limited saturated output power of the power amplifier $(29 \mathrm{dBm})$, the tripler circuit could not be driven into saturation and therefore the maximum output power and efficiency were not reached.

\section{CONCLUSION}

We have demonstrated a silicon integrated HBV frequency tripler. The performance of the presented device is comparable with the state-of-the-art hybrid and monolithically integrated InP-based frequency tripler devices [15]. The InGaAs/ InAlAs/ AlAs material structure was transferred onto silicon using LT plasma assisted bonding. By use of alloyed ohmic contact is a reason improved RF performance in comparison with the devices reported in [21].

The presented results motivate further research and development of III-V HBV devices integrated on silicon for $\mathrm{THz}$ frequencies. Silicon micromachining can be utilized to 
form membranes, waveguides, and antennas for more advanced integration schemes.

\section{ACKNOWLEDGMENT}

Authors would like to acknowledge Dr. Z. Lai from Nanofabrication Laboratory, Department of Microtechnology and Nanoscience, Chalmers University of Technology for TEM images and wish to thank Dr. A. Ø. Olsen from Wasa Millimeter Wave AB for assistance with RF measurements.

\section{REFERENCES}

[1] P. Siegel, "Terahertz technology," IEEE Trans. Microw. Theory Techn., vol. 50, pp. 910-927, 2002.

[2] G. Chattopadhyay, "Technology capabilities, and performance of low power terahertz sources," IEEE Trans. THz Sci. Technol., vol. 1, pp. 3353, 2011.

[3] M. Lapisa, G. Stemme, and F. Niklaus, "Wafer-level heterogeneous integration for MOEMS, MEMS and NEMS," IEEE J. Sel. Topics Quantum Electron., vol. 17, pp. 629-644, 2011.

[4] V.M. Lubecke, K. Mizuno, and G.M. Rebeiz, "Micromachining for terahertz applications," IEEE Trans. Microw. Theory Techn., vol. 46, pp. 1821-1831, 1998.

[5] P. L. Kirby, Y. Li, Q. Xiao, J. L. Hesler, and J. Papapolymerou, "Silicon Micromachined Multiplier Utilizing Heterostructure Barrier Varactor Diode " in IMS, 2007, pp. 1141 - 1144.

[6] O. Moutanabbir and U. Gösele, "Heterogenous integration of compound semiconductors," Ann. Revi. Mater. Res., vol. 40, pp. 469-500, 2010.

[7] K. Eisenbeiser, et al., "GaAs MESFETs fabricated on Si substrates using a SrTiO3 buffer layer," IEEE Electron Device Lett., vol. 23, pp. 300-302, 2002.

[8] K.L. Lew, et al., "InGaP/GaAs heterojunction bipolar transistor grown on Si substrate with SiGe graded buffer layer," Electronics Lett., vol. 44, pp. 243-244, 2008.

[9] T. Kraemer, C. Meliani, F.J. Schmueckle, J. Wuerfl, and G. Traenkle, "Travelling-wave amplifiers in transferred substrate InP-HBT technology," IEEE Trans. Microw. Theory Techn., vol. 57, pp. 21142121, 2009.

[10] J.W. Chung, J. Lee, E.L. Piner, and T. Palacios, "Seamless On-Wafer Integration of $\mathrm{Si}(100)$ MOSFETs and GaN HEMTs," IEEE Electron Device Lett., vol. 30, pp. 1015-1017, 2009.

[11] S. Arscott, P. Mounaix, and D. Lippens, "Substrate transfer process for InP-based heterostrucutre barrier varactor devices," J. Vac. Sci. Technol. $B$, vol. 18, pp. 150-155, 2000.

[12] S.M. Marazita, et al., "Integrated GaAs Schottky mixers by spin-on-glass dielectric wafer bonding," IEEE Trans. Electron Devices, vol. 47, pp. 1152-1157, 2000.

[13] D. Pasquariello and K. Hjort, "Plasma-assisted InP-to-Si temperature wafer bonding," IEEE J. Sel. Topics Quantum Electron., vol. 8, pp. 118$131,2002$.

[14] J. Vukusic, et al., "HBV tripler with $21 \%$ efficiency at $102 \mathrm{GHz}$," Electronic Lett., vol. 42, pp. 355-356, 2006.

[15] A. Malko, T. Bryllert, J. Vukusic, and J. Stake, "High efficiency and broad-band operation of monolithically integrated W-band HBV frequency tripler," presented at the 24th IPRM, 2012.

[16] E. Kollberg and A. Rydberg, "Quantum-barrier-varactor diodes for highefficiency millimetre-wave multipliers," Electronics Lett., vol. 25, pp. 1696-1698, 1989

[17] T. A. Emadi, T. Bryllert, M. Sadeghi, J. Vukusic, and J. Stake, "Optimum barrier thickness studies for InGaAs/InAlAs/AlAs heterostrucutre barrier varactor diode," Appl. Phys. Lett., vol. 90, pp. 012108 - 012108-3 2007.

[18] J. Stake, S. H. Jones, L. Dillner, S. Hollung, and E. Kollberg, "Heterostrucutre-barrier-varactor design," IEEE Trans. Microw. Theory Techn., vol. 48, pp. 677-682, 2000.

[19] M. H. Tavakoli Dastjerdi, A. Sanz-Velasco, J. Vukusic, M. Sadeghi, and J. Stake, "InGaAs/InAlAs/AlAs heterostructure barrier varactors on silicon substrate," IEEE Electron Device Lett., vol. 32, pp. 140 - 142, 2010.

[20] J. Vukusic, T. Bryllert, A. Ø. Olsen, and J. Stake, "High-power W-band monolithically integrated tripler," in 34th IRMMW-THz, 2009, pp. 1-2.
[21] A. Malko, T. Bryllert, J. Vukusic, and J. Stake, "Integrated III-V heterostructure barrier varactor frequency tripler on a silicon substarte," in 7th EuMIC, 2012, pp. 1-4. 